\title{
Desempenho agronômico de seleções de café Bourbon Vermelho e Bourbon Amarelo de diferentes origens
}

\author{
André Dominghetti Ferreira(1), Gladyston Rodrigues Carvalho(2), Juliana Costa de Rezende(2), \\ Cesar Elias Botelho( ${ }^{(2)}$, Ramiro Machado Rezende ${ }^{(3)}$ e Alex Mendonça de Carvalho(3)
}

\begin{abstract}
(1)Embrapa Gado de Corte, Av. Rádio Maia, № 830, CEP 79106-550 Campo Grande, MS. E-mail: andre.dominghetti@embrapa.br (2)Empresa de Pesquisa Agropecuária de Minas Gerais, Unidade Regional do Sul de Minas, Campus da Universidade Federal de Lavras, s/no, Caixa Postal 176, CEP 37200-000 Lavras, MG. E-mail: carvalho@epamig.ufla.br, julianacosta@epamig.br, cesarbotelho@epamig.br (3)Universidade Federal de Lavras, Departamento de Fitotecnia, Caixa Postal 3037, CEP 37200-000 Lavras, MG. E-mail: ramiromr@globo.com, carvalho.am@hotmail.com
\end{abstract}

Resumo - O objetivo deste trabalho foi avaliar a adaptabilidade e estabilidade fenotípica e outras características de interesse agronômico de genótipos de café Bourbon Vermelho e Bourbon Amarelo, para selecionar os de melhor desempenho no Estado de Minas Gerais. Foram avaliados 17 genótipos pertencentes ao grupo Bourbon, bem pontuados em concursos de qualidade de bebida, além de três cultivares amplamente cultivadas no estado, utilizadas como testemunhas. Os experimentos foram instalados em dezembro de 2005, nos municípios de Lavras, Santo Antônio do Amparo, Três Pontas, Campos Altos e Patrocínio. As avaliações foram realizadas durante as quatro primeiras colheitas, nos anos agrícolas 2007/2008, 2008/2009, 2009/2010 e 2010/2011, e compreenderam as seguintes características: produtividade de grãos, adaptabilidade e estabilidade fenotípica, percentagem de frutos chochos, vigor vegetativo e classificação por peneira. Há variabilidade genética dentro do grupo de Bourbon estudado. Os genótipos de Bourbon apresentam produtividades satisfatórias em todos os locais avaliados. $\mathrm{O}$ genótipo Bourbon Vermelho 2 apresenta maior adaptabilidade, tendo-se destacado quanto a todas as características avaliadas.

Termos para indexação: Coffea arabica, adaptabilidade, estabilidade, genótipo x ambiente, qualidade de bebida.

\section{Agronomic performance of selections of Red Bourbon and Yellow Bourbon coffee from different origins}

\begin{abstract}
The objective of this work was to evaluate phenotypic adaptability and stability, and other traits of agronomic interest of genotypes of Red Bourbon and Yellow Bourbon coffee in order to select the ones with better performance in the state of Minas Gerais, Brazil. We evaluated 17 genotypes of the Bourbon cultivar group, with good performance in cup-quality tests, as well as three cultivars widely grown in the state, used as a control. The experiments were established in December 2005, in the municipalities of Lavras, Santo Antônio do Amparo, Três Pontas, Campos Altos, and Patrocínio. The evaluations were done during the first four harvests in the growing seasons of 2007/2008, 2008/2009, 2009/2010, and 2010/2011, including the following traits: grain yield, phenotypic adaptability and stability, percentage of floating grains, vegetative vigor, and bean size. There is genetic variability within the Bourbon group studied. Bourbon genotypes show satisfactory yields in all evaluated sites. The genotype Red Bourbon 2 has greater adaptability, and stood out as to all studied variables.
\end{abstract}

Index terms: Coffea arabica, adaptability, stability, genotype x environment, coffee cup-quality.

\section{Introdução}

Um dos principais instrumentos que as empresas rurais e as organizações ligadas à cafeicultura dispõem para ingressar no mercado de café é a qualidade. Em decorrência da crescente demanda por cafés com alta qualidade de bebida, o interesse no plantio de cultivares de maior potencial para produção de cafés especiais tem aumentado significativamente nos últimos anos, o que leva os pesquisadores a darem ênfase não só à produtividade das cultivares.

Entre as cultivares comerciais do cafeeiro (Coffea arabica L.) disponíveis para o plantio, a cultivar Bourbon apresenta elevado potencial quanto à qualidade de bebida e é altamente valorizada nos mercados de cafés especiais, por suas características sensoriais diferenciadas. No entanto, sua adoção pelos produtores tem sido lenta, principalmente em razão 
da falta de informações sobre seu comportamento agronômico nos diferentes sistemas de produção utilizados na cafeicultura nacional.

De maneira geral, as plantas da cultivar Bourbon são menos produtivas que as da cultivar Mundo Novo (Fazuoli et al., 2005); porém, a adaptação de genótipos a ambientes específicos pode consistir a diferença entre uma boa e uma excelente variedade. Vasconcelos et al. (2010) ressaltam que interações significativas associadas a características ambientais previsíveis representam uma grande oportunidade de exploração. Uma das formas mais utilizadas para determinar o efeito dessa interação é a implantação de experimentos em locais contrastantes, em que vários genótipos são avaliados, com vistas à identificação de materiais genéticos com ampla estabilidade e adaptabilidade (Barros et al., 2012; Mendes et al., 2012; Silveira et al., 2012).

No método proposto por Annicchiarico (1992), a estabilidade é medida pela superioridade de um genótipo em relação ao desempenho médio dos genótipos em cada ambiente. Esse método é baseado no cálculo de um índice de recomendação ou confiança, de tal forma que os maiores valores do índice são obtidos para aqueles genótipos que apresentam maior média percentual e menor desvio (Ramos et al., 2011). Condé et al. (2010) e Pereira et al. (2012) recomendam a utilização dessa metodologia pela facilidade de interpretação.

O objetivo deste trabalho foi avaliar a adaptabilidade e estabilidade fenotípica e outras características de interesse agronômico de genótipos de café Bourbon Vermelho e Bourbon Amarelo.

\section{Material e Métodos}

Foram avaliados 20 genótipos de café, dos quais 17 pertencem ao grupo da cultivar Bourbon, obtidos em lavouras que tiveram seus produtos bem pontuados em concursos de qualidade de bebida. Essas fazendas estão localizadas em regiões tradicionais de cultivo do café no Estado de Minas Gerais, mas não têm registro sobre a origem da cultivar plantada inicialmente. Portanto, as cultivares foram denominadas pelo nome da fazenda: Bourbon Amarelo LCJ 10 Fazenda Experimental da Epamig de Machado, MG (BA1), Bourbon Amarelo Fazenda Experimental do Procafé (BA2), Bourbon Amarelo Fazenda Bom Jardim (BA3), Bourbon Amarelo Fazenda Betânia (BA4), Bourbon Amarelo Fazenda Boa Vista (BA5), Bourbon Amarelo
LCJ 9 (IAC, SP) (BA6), Bourbon Amarelo Fazenda Toriba (BA7), Bourbon Amarelo Fazenda São Paulo (BA8), Bourbon Amarelo Fazenda Castro (BA9), Bourbon Amarelo Fazenda Nogueira (BA10), Bourbon Amarelo Fazenda Paixão (BA11), Bourbon Amarelo Fazenda Samambaia (BA12), Bourbon Amarelo Italiano Fazenda Monte Alegre (BA13), Bourbon Amarelo Trigo Fazenda Monte Alegre (BA14), Bourbon Amarelo Limoeiro Fazenda Monte Alegre (BA15), Bourbon Vermelho Fazenda Experimental do Procafé (BV1) e Bourbon Vermelho Fazenda São João Batista (BV2). As cultivares Mundo Novo IAC 502/9, Catuaí Vermelho IAC 144 e Icatú Amarelo IAC 3282, amplamente cultivadas no Estado de Minas Gerais, foram utilizadas como testemunhas.

Os experimentos foram instalados em dezembro de 2005 no espaçamento de $3,5 \times 0,8 \mathrm{~m}$, nos municípios de Lavras, Santo Antônio do Amparo, Três Pontas, Campos Altos e Patrocínio, de forma a representar as condições de ambiente existentes nas regiões direcionadas para produção de cafés finos (Tabela 1). Utilizou-se o delineamento de blocos ao acaso, com três repetições e dez plantas por parcela, sendo as oito centrais utilizadas para a coleta dos frutos. A correção e fertilização do solo, bem como o controle de pragas e doenças, foram iguais em todos os locais, com o intuito de fornecer a mais alta tecnologia disponível no sistema de produção.

As avaliações foram realizadas nas quatro primeiras colheitas, durante os anos agrícolas 2007/2008, 2008/2009, 2009/2010 e2010/2011, tendo compreendido as seguintes características: produtividade de grãos, adaptabilidade e estabilidade fenotípica, percentagem de frutos chochos, vigor vegetativo e classificação por

Tabela 1. Região e caracterização geográfica dos locais de instalação dos experimentos no Estado de Minas Gerais.

\begin{tabular}{lccccc}
\hline Variável & \multicolumn{5}{c}{ Municípios } \\
\cline { 2 - 6 } & CA & SAA & Patrocínio & Lavras & TP \\
\hline Região & Alto & Campo das & Alto & Sul & Sul \\
& Paranaíba & Vertentes & Paranaíba & & \\
Altitude (m) & 1.230 & 960 & 966 & 919 & 905 \\
$\mathrm{~T}_{\text {Média }}\left({ }^{\circ} \mathrm{C}\right)$ & 17,6 & 19,8 & 22,0 & 19,3 & 18,0 \\
$\mathrm{P}_{\text {Anual }}(\mathrm{mm})$ & 1.830 & 1.670 & 1.620 & 1.529 & 1.545 \\
Latitude & $19^{\circ} 41^{\prime} 46^{\prime \prime} \mathrm{S}$ & $20^{\circ} 56^{\prime} 47^{\prime \prime} \mathrm{S}$ & $18^{\circ} 56^{\prime} 38^{\prime \prime} \mathrm{S}$ & $21^{\circ} 14^{\prime} 43^{\prime \prime} \mathrm{S}$ & $21^{\circ} 20^{\prime} 50^{\prime \prime} \mathrm{S}$ \\
Longitude & $46^{\circ} 59^{\prime} 33^{\prime \prime} \mathrm{N}$ & $44^{\circ} 55^{\prime} 08^{\prime \prime} \mathrm{O}$ & $46^{\circ} 59^{\prime} 33^{\prime \prime} \mathrm{N}$ & $44^{\circ} 59^{\prime} 59^{\prime \prime} \mathrm{O}$ & $45^{\circ} 28^{\prime} 23^{\prime \prime O}$ \\
Região & Cerrado & Sul de & Cerrado & Sul de & Sul de \\
cafeeira & de Minas & Minas & de Minas & Minas & Minas \\
\hline
\end{tabular}

CA, Campos Altos; SAA, Santo Antônio do Amparo; TP, Três Pontas; T, temperatura; $\mathrm{P}_{\text {Anual, }}$, precipitação anual. 
peneira. As colheitas foram realizadas em várias etapas, para se obter, no mínimo, $80 \%$ de frutos no estádio cereja em cada parcela.

A produção foi medida pela pesagem dos frutos, imediatamente após a colheita. Posteriormente, foi realizada a conversão para sacas de $60 \mathrm{~kg}$ de café beneficiado por hectare, por meio do rendimento de uma amostra de $3 \mathrm{~kg}$ de café, coletada em cada parcela por ocasião da colheita. A percentagem de frutos chochos foi determinada pela metodologia proposta por Antunes Filho \& Carvalho (1957), em que são colocados 100 frutos cereja em água e são considerados chochos aqueles que permanecem na superfície.

Para avaliar o vigor vegetativo dos cafeeiros, foram atribuídas notas de acordo com escala arbitrária de 10 pontos, em que a nota 1 correspondente às piores plantas, com reduzido vigor vegetativo e acentuado sintoma de depauperamento; e a nota 10 , às plantas com excelente vigor, mais enfolhadas e com acentuado crescimento vegetativo dos ramos produtivos, conforme Carvalho et al. (1979). Para a classificação dos grãos, passou-se uma amostra de 300 g por um conjunto de peneiras intercaladas (12/64 a 19/64). A massa dos grãos retidos em cada peneira foi quantificada e transformada em percentagem (Brasil, 2003).

A análise de variância foi feita com arranjo conjunto das quatro colheitas, dos cinco locais avaliados (Steel \& Torrie, 1980), após constatação da homogeneidade da variância, pelo teste de Hartley, conforme sugerido por Ramalho et al. (2000). Em seguida, as médias foram comparadas pelo teste de Scott-Knott, a 5\% de probabilidade. Utilizou-se o aplicativo computacional Sisvar.

Após constatação da significância da interação progênies $\mathrm{x}$ biênios $\mathrm{x}$ locais, foi realizada análise da adaptabilidade e da estabilidade das cultivares, tendose considerado, como ambientes, as combinações de biênios com locais. Foi utilizada a metodologia proposta por Annicchiarico (1992), o qual propõe o uso de um índice de confiança que estima o risco da adoção de determinado genótipo. Os procedimentos para os cálculos, pelo método proposto, iniciaram-se com a transformação das médias de produtividade de cada cultivar, em cada ambiente, em percentagem da média do ambiente. Posteriormente, estimou-se a média $\mathrm{Y}_{\mathrm{i}(\mathrm{GFD})}$ e o desvio-padrão $\left(\mathrm{S}_{\mathrm{i}(\mathrm{GFD})}\right)$ das percentagens de cada cultivar para os ambientes de modo geral $(\mathrm{G})$ e para os ambientes favoráveis (F) e desfavoráveis (D). Utilizouse o aplicativo computacional Genes (Cruz, 2006).

\section{Resultados e Discussão}

Houve efeito significativo na interação genótipo $\mathrm{x}$ local para todas as características avaliadas, o que indica comportamento distinto dos genótipos nos ambientes estudados. Esses resultados refletem as diferentes sensibilidades dos genótipos de cafeeiro às mudanças do ambiente, o que também foi verificado por outros autores (Botelho et al., 2010; Cilas et al., 2010; Gichimu \& Omondi, 2010).

A produtividade de grãos dos genótipos foi diferente apenas nos experimentos instalados em Santo Antônio do Amparo e Patrocínio, e não foi observada diferença entre os genótipos nos demais locais de cultivo (Tabela 2). Nesses dois locais, houve formação de três grupos de genótipos com produtividades distintas. Dos genótipos avaliados, apenas os Bourbon Amarelo 5, 10 e 14, bem como a cultivar Icatú Amarelo IAC 3282, compuseram o grupo de menor produtividade.

O grupo de maior produtividade em Santo Antônio do Amparo, formado por 12 genótipos,

Tabela 2. Produtividade de grãos de 20 genótipos de café arábica, avaliados por quatro anos em cinco municípios do Estado de Minas Gerais ${ }^{(1)}$.

\begin{tabular}{|c|c|c|c|c|c|c|}
\hline \multirow[t]{2}{*}{ Genótipo $^{(2)}$} & $\begin{array}{c}\text { Campos } \\
\text { Altos }\end{array}$ & $\mathrm{SAA}^{(3)}$ & $\begin{array}{l}\text { Patro- } \\
\text { cínio }\end{array}$ & Lavras & $\begin{array}{c}\text { Três } \\
\text { Pontas }\end{array}$ & Média \\
\hline & \multicolumn{6}{|c|}{ 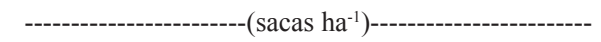 } \\
\hline BA1 & $24,67 \mathrm{a}$ & $56,74 \mathrm{a}$ & $29,41 \mathrm{c}$ & $25,89 \mathrm{a}$ & $26,51 \mathrm{a}$ & $32,64 a$ \\
\hline BA2 & $22,71 \mathrm{a}$ & $52,89 a$ & $28,94 \mathrm{c}$ & $24,31 \mathrm{a}$ & $20,76 a$ & $29,92 \mathrm{a}$ \\
\hline BA3 & $23,63 a$ & $49,87 \mathrm{a}$ & $31,21 \mathrm{c}$ & $23,87 \mathrm{a}$ & $24,99 a$ & $30,71 \mathrm{a}$ \\
\hline BA4 & $23,55 \mathrm{a}$ & $34,47 b$ & $27,32 \mathrm{c}$ & $24,74 \mathrm{a}$ & $21,85 \mathrm{a}$ & $26,39 b$ \\
\hline BA5 & $27,22 \mathrm{a}$ & $28,00 \mathrm{c}$ & $28,68 \mathrm{c}$ & $25,13 a$ & $22,17 \mathrm{a}$ & $26,24 b$ \\
\hline BA6 & $23,84 a$ & $54,00 \mathrm{a}$ & $24,97 \mathrm{c}$ & $27,12 \mathrm{a}$ & $17,02 \mathrm{a}$ & $29,39 a$ \\
\hline BA7 & $23,15 \mathrm{a}$ & $55,74 \mathrm{a}$ & $31,01 \mathrm{c}$ & $25,26 \mathrm{a}$ & $19,47 \mathrm{a}$ & $30,93 \mathrm{a}$ \\
\hline BA8 & $24,55 \mathrm{a}$ & $45,96 a$ & $27,57 \mathrm{c}$ & $30,57 \mathrm{a}$ & $20,78 \mathrm{a}$ & $29,89 \mathrm{a}$ \\
\hline BA9 & $15,81 \mathrm{a}$ & $42,90 \mathrm{a}$ & $30,13 \mathrm{c}$ & $32,81 \mathrm{a}$ & $19,87 \mathrm{a}$ & $28,30 \mathrm{a}$ \\
\hline BA10 & $22,86 \mathrm{a}$ & $21,25 \mathrm{c}$ & $30,78 \mathrm{c}$ & $27,10 \mathrm{a}$ & $22,43 a$ & $24,88 \mathrm{~b}$ \\
\hline BA11 & $17,72 \mathrm{a}$ & $50,10 \mathrm{a}$ & $26,74 \mathrm{c}$ & $22,00 \mathrm{a}$ & $16,19 \mathrm{a}$ & $26,55 b$ \\
\hline BA12 & $16,25 \mathrm{a}$ & $50,87 \mathrm{a}$ & $27,65 \mathrm{c}$ & $27,39 a$ & $20,46 a$ & $28,52 \mathrm{a}$ \\
\hline BV1 & $24,29 a$ & $35,74 b$ & $16,93 \mathrm{c}$ & $24,51 \mathrm{a}$ & $19,75 \mathrm{a}$ & $24,24 b$ \\
\hline BV2 & $27,51 \mathrm{a}$ & $47,81 \mathrm{a}$ & $43,64 b$ & $32,67 \mathrm{a}$ & $25,25 \mathrm{a}$ & $35,38 \mathrm{a}$ \\
\hline BA13 & $34,87 \mathrm{a}$ & $21,28 \mathrm{c}$ & $40,60 b$ & $31,87 \mathrm{a}$ & $21,29 a$ & $29,98 \mathrm{a}$ \\
\hline BA14 & $24,68 \mathrm{a}$ & $29,74 \mathrm{c}$ & $31,01 \mathrm{c}$ & $32,54 \mathrm{a}$ & $23,00 \mathrm{a}$ & $28,19 \mathrm{a}$ \\
\hline BA15 & $28,32 \mathrm{a}$ & $21,46 \mathrm{c}$ & $56,41 \mathrm{a}$ & $32,63 \mathrm{a}$ & $22,89 a$ & $32,34 \mathrm{a}$ \\
\hline Mundo Novo ${ }^{(4)}$ & $25,31 a$ & $54,60 \mathrm{a}$ & $35,47 \mathrm{c}$ & $33,12 \mathrm{a}$ & $26,78 \mathrm{a}$ & $35,06 a$ \\
\hline Catuaí Vermelho(4) & $27,15 \mathrm{a}$ & $47,41 \mathrm{a}$ & $34,14 \mathrm{c}$ & $29,82 \mathrm{a}$ & $24,61 \mathrm{a}$ & $32,63 \mathrm{a}$ \\
\hline Icatú Amarelo(4) & $23,51 \mathrm{a}$ & $37,72 b$ & $42,32 b$ & $27,25 \mathrm{a}$ & $25,63 a$ & $31,29 a$ \\
\hline
\end{tabular}

${ }^{(1)}$ Médias seguidas de letras iguais, nas colunas, não diferem pelo teste de Scott-Knott, a $5 \%$ de probabilidade. ${ }^{(2)} \mathrm{BA}$, Bourbon Amarelo; BV, Bourbon Vermelho. ${ }^{(3)}$ SAA, Santo Antônio do Amparo. ${ }^{(4)}$ Testemunhas: Mundo Novo IAC 502/9, Catuaí Vermelho IAC 144 e Icatú Amarelo IAC 3282. 
obteve médias que variaram de 42,90 a 56,74 sacas por hectare. Em Patrocínio, o genótipo Bourbon Amarelo 15 foi superior a todos os demais, e o grupo de menor produtividade foi constituído pela maioria dos genótipos. Com base na média geral de quatro colheitas, houve a formação de dois grupos, em que a produtividade variou entre 28,19 e 35,38 sacas por hectare no grupo superior, composto por 15 cultivares, entre elas as cultivares utilizadas como testemunhas (Tabela 2).

Em ensaio conduzido por 33 anos em Campinas, SP, Fazuoli et al. (2005) verificaram que as progênies de Mundo Novo foram superiores às de Bourbon Amarelo e de Bourbon Vermelho em 38,7 e 111,6\%, respectivamente, sendo que, entre as 30 progênies mais produtivas, não houve nenhuma progênie de Bourbon. Contudo, no presente trabalho, as cultivares de Bourbon utilizadas apresentaram produtividades satisfatórias em todos os locais avaliados, com valores iguais ou superiores aos da cultivar Mundo Novo. Esse resultado pode ser atribuído ao maior uso de tecnologia no sistema de produção utilizado no presente trabalho, que possibilitou a expressão do potencial produtivo dessas cultivares. Dessa forma, nota-se que os genótipos em estudo são competitivos em relação aos comumente utilizados, principalmente com o emprego de técnicas de cultivo mais intensivas.

Na literatura, recomenda-se a avaliação da produção por pelo menos quatro safras consecutivas, ou dois biênios, para ter sucesso na seleção de uma progênie, visto que o café é uma cultura perene e a estabilidade de produção é alcançada na quarta colheita (Pedro et al., 2011). Portanto, o ciclo de avaliação utilizado no presente trabalho foi suficiente para discriminar, com eficiência, o potencial produtivo das progênies.

O comportamento distinto das cultivares estudadas nos diferentes locais de cultivo confirma a ocorrência da interação genótipo $\mathrm{x}$ ambiente e justifica a avaliação de estabilidade dos genótipos (Tabela 3). No presente trabalho, os genótipos Bourbon Vermelho 2 e Mundo Novo 502/9 apresentaram maiores valores do índice de confiança (Annicchiarico, 1992), que foram de 105,32 e 98,74, respectivamente. O genótipo Bourbon Vermelho 2 apresentou o melhor desempenho, com 90\% de índice de confiança, 5,32\% mais produtivo que a média ambiental, o que confirma os resultados obtidos na Tabela 2, em que esse genótipo esteve nos grupos de maior produtividade nos diferentes locais.
Portanto, essa cultivar de Bourbon e a cultivar Mundo Novo 502/9 se mostraram promissoras, pois além de apresentarem maior estabilidade nos ambientes, elas figuraram entre as mais produtivas na média dos ambientes. O genótipo Bourbon Amarelo 10, por sua vez, foi o que apresentou menor valor do índice de confiança, com risco de $40,09 \%$ de se comportar abaixo da média dos ambientes.

Ao se compararem os desempenhos desses dois genótipos de Bourbon, nos ambientes mais desfavoráveis para cada um, o Bourbon Vermelho 2 produzirá $45,41 \%$ a mais que o Bourbon Amarelo 10 , em relação à média ambiental. Ao se considerar a média de produtividade mineira, em torno de 24,6 sacas por hectare (Companhia Nacional de Abastecimento, 2013), haveria um aumento de 11,17 sacas por hectare com a escolha correta da cultivar.

Observou-se, para todos os genótipos e em todos os locais de estudo, maior percentagem de frutos granados em relação aos chochos, com média geral de $92,96 \%$, para frutos granados, e de $7,04 \%$ para frutos chochos (Tabela 4). Segundo Carvalho et al. (2006), acima de $90 \%$ de frutos bem granados é um percentual considerado satisfatório pelos

Tabela 3. Estimativas da média de produção em quatro anos $\left(\mathrm{Y}_{\mathrm{i}}\right.$, sacas ha-1 ${ }^{-1}$, dos desvios $\left(\mathrm{S}_{\mathrm{i}}\right)$ e do índice de confiança $\left(\mathrm{I}_{\mathrm{i}}\right)$ segundo o método proposto por Annicchiarico (1992) ${ }^{(1)}$.

\begin{tabular}{lccc}
\hline Genótipo $^{(2)}$ & $\mathrm{Y}_{\mathrm{i}}$ & $\mathrm{S}_{\mathrm{i}}$ & $\mathrm{I}_{\mathrm{i}}^{\left({ }^{(1)}\right.}$ \\
\hline BA1 & 108,72 & 22,14 & 79,51 \\
BA2 & 98,99 & 16,98 & 76,45 \\
BA3 & 102,13 & 15,01 & 84,32 \\
BA4 & 91,84 & 13,21 & 75,56 \\
BA5 & 89,51 & 18,12 & 68,36 \\
BA6 & 94,83 & 22,58 & 66,01 \\
BA7 & 103,98 & 17,62 & 84,34 \\
BA8 & 102,45 & 11,54 & 88,00 \\
BA9 & 94,94 & 23,15 & 66,19 \\
BA10 & 86,71 & 21,45 & 59,91 \\
BA11 & 89,96 & 18,59 & 65,62 \\
BA12 & 93,21 & 22,26 & 65,01 \\
BV1 & 87,02 & 16,01 & 67,21 \\
BV2 & 118,87 & 9,52 & 105,32 \\
BA13 & 110,87 & 37,85 & 65,21 \\
BA14 & 104,00 & 19,23 & 78,15 \\
BA15 & 115,23 & 39,69 & 91,99 \\
\hline Mundo Novo & $13)$ & 11,45 & 98,74 \\
Catuaí Vermelho & 101,40 & 10,09 & 90,24 \\
Icatú Amarelo A $^{(3)}$ & 109,32 & 24,54 & 79,37 \\
\hline
\end{tabular}

${ }^{(1)}$ Nível de significância adotado $=0,10 .{ }^{(2)} \mathrm{BA}$, Bourbon Amarelo; BV, Bourbon Vermelho. ${ }^{(3)}$ Testemunhas: Mundo Novo IAC 502/9, Catuaí Vermelho IAC 144 e Icatú Amarelo IAC 3282. 
pesquisadores na avaliação e seleção de cafeeiros em programa de melhoramento, uma vez que grande parte das cultivares comerciais apresenta este percentual.

Em Campos Altos, houve a formação de dois grupos, e os genótipos de Bourbon Amarelo 13, 14 e 15 , todos provenientes de uma mesma fazenda, foram iguais entre si e superiores aos demais (Tabela 4). Semelhantemente, em Santo Antônio do Amparo, houve a formação de dois grupos; porém, neste local, a maior parte dos genótipos tiveram notas superiores, e os genótipos de Bourbon Amarelo 4, 5, 10 e 11 apresentaram notas inferiores.

É possível verificar a influência de fatores genéticos e ambientais sobre percentagem de frutos granados, pela variação entre os genótipos e entre os locais estudados. Vale destacar que apenas o genótipo Bourbon Vermelho 1 permaneceu no grupo de maior percentagem de grãos chochos, nos três locais onde foi detectada diferença entre os genótipos, o que indica possível causa genética. Outros autores também

Tabela 4. Percentagem de grãos chochos nos 20 genótipos de café, avaliados por quatro anos em cinco municípios do Estado de Minas Gerais ${ }^{(1)}$.

\begin{tabular}{|c|c|c|c|c|c|}
\hline Genótipo $^{(2)}$ & $\begin{array}{c}\text { Campos } \\
\text { Altos }\end{array}$ & $\mathrm{SAA}^{(3)}$ & Patrocínio & Lavras & $\begin{array}{c}\text { Três } \\
\text { Pontas }\end{array}$ \\
\hline & & & & & \\
\hline BA1 & $6,67 a$ & $11,00 \mathrm{a}$ & $6,00 \mathrm{a}$ & $10,00 \mathrm{~b}$ & $2,00 \mathrm{a}$ \\
\hline BA2 & $6,33 a$ & $10,33 \mathrm{a}$ & $4,67 \mathrm{a}$ & $9,33 b$ & $4,00 \mathrm{a}$ \\
\hline BA3 & $4,67 \mathrm{a}$ & $9,67 \mathrm{a}$ & $6,67 \mathrm{a}$ & $9,67 \mathrm{~b}$ & $5,33 \mathrm{a}$ \\
\hline BA4 & $6,00 \mathrm{a}$ & $10,00 \mathrm{a}$ & $6,67 \mathrm{a}$ & $12,67 b$ & $8,33 b$ \\
\hline BA5 & $3,00 \mathrm{a}$ & $12,00 \mathrm{a}$ & $3,67 \mathrm{a}$ & $8,33 b$ & $6,33 \mathrm{a}$ \\
\hline BA6 & $6,00 \mathrm{a}$ & $9,33 \mathrm{a}$ & $4,00 \mathrm{a}$ & $9,67 b$ & $4,67 \mathrm{a}$ \\
\hline BA7 & $4,00 \mathrm{a}$ & $8,33 a$ & $6,33 a$ & $10,00 \mathrm{~b}$ & $4,00 \mathrm{a}$ \\
\hline BA8 & $5,67 \mathrm{a}$ & $9,00 \mathrm{a}$ & $5,00 \mathrm{a}$ & $6,67 \mathrm{a}$ & $3,67 \mathrm{a}$ \\
\hline BA9 & $3,33 \mathrm{a}$ & $9,00 \mathrm{a}$ & $5,00 \mathrm{a}$ & $9,33 b$ & $4,67 \mathrm{a}$ \\
\hline BA10 & $6,00 \mathrm{a}$ & $15,00 \mathrm{a}$ & $7,33 \mathrm{a}$ & $11,00 \mathrm{~b}$ & $8,67 b$ \\
\hline BA11 & $10,67 \mathrm{a}$ & $12,00 \mathrm{a}$ & $3,67 \mathrm{a}$ & $11,00 \mathrm{~b}$ & $8,00 \mathrm{~b}$ \\
\hline BA12 & $5,00 \mathrm{a}$ & $8,67 \mathrm{a}$ & $6,67 \mathrm{a}$ & $8,33 b$ & $5,00 \mathrm{a}$ \\
\hline BV1 & $4,67 \mathrm{a}$ & $11,00 \mathrm{a}$ & $14,33 b$ & $8,67 b$ & $10,67 b$ \\
\hline BV2 & $6,00 \mathrm{a}$ & $8,67 \mathrm{a}$ & $8,00 \mathrm{a}$ & $8,67 b$ & $6,00 \mathrm{a}$ \\
\hline BA13 & $2,33 a$ & $8,00 \mathrm{a}$ & $4,33 \mathrm{a}$ & $6,33 a$ & $4,67 \mathrm{a}$ \\
\hline BA14 & $2,67 \mathrm{a}$ & $8,33 \mathrm{a}$ & $4,00 \mathrm{a}$ & $4,00 \mathrm{a}$ & $5,33 \mathrm{a}$ \\
\hline BA15 & $3,33 \mathrm{a}$ & $10,33 \mathrm{a}$ & $5,67 \mathrm{a}$ & $7,67 \mathrm{a}$ & $4,33 \mathrm{a}$ \\
\hline Mundo Novo ${ }^{(4)}$ & $5,67 \mathrm{a}$ & $9,00 \mathrm{a}$ & $7,67 \mathrm{a}$ & $6,33 a$ & $4,00 \mathrm{a}$ \\
\hline Catuaí Vermelho ${ }^{(4)}$ & $3,67 \mathrm{a}$ & $6,33 a$ & $6,67 \mathrm{a}$ & $4,67 \mathrm{a}$ & $3,67 \mathrm{a}$ \\
\hline Icatú Amarelo $^{(4)}$ & $6,00 \mathrm{a}$ & $8,67 \mathrm{a}$ & $4,67 \mathrm{a}$ & $14,00 \mathrm{~b}$ & $7,00 \mathrm{~b}$ \\
\hline
\end{tabular}

${ }^{(1)}$ Médias seguidas de letras iguais, nas colunas, não diferem pelo teste de Scott-Knott, a 5\% de probabilidade. ${ }^{(2)} \mathrm{BA}$, Bourbon Amarelo; BV, Bourbon Vermelho. ${ }^{(3)}$ SAA, Santo Antônio do Amparo. ${ }^{(4)}$ Testemunhas: Mundo Novo IAC 502/9, Catuaí Vermelho IAC 144 e Icatú Amarelo IAC 3282. observaram variabilidade para essa característica em ensaios de melhoramento genético. Ao estudar progênies de Mundo Novo, Antunes Filho \& Carvalho (1957) encontraram percentuais de sementes chochas que variaram de 0 a $80 \%$; já Carvalho et al. (2006), ao avaliar progênies de Coffea arabica, verificaram, para esta característica, uma amplitude de variação de 4,5 a 18,25\%, atribuída à variabilidade genética.

As médias de vigor do experimento instalado em Patrocínio foram as mais baixas, tendo-se formado três grupos distintos (Tabela 5). Os resultados permitem inferir que os genótipos de Bourbon, cultivados sob o sistema de sequeiro, não apresentam boa adaptabilidade nesta região, o que evidencia a influência ambiental sobre o desenvolvimento das plantas e exige maiores cuidados na condução de lavouras com essas cultivares, visto que um elevado vigor vegetativo está correlacionado positivamente à melhor adaptação da cultivar ao ambiente de cultivo (Severino et al., 2002).

No ensaio instalado em Três Pontas, houve a formação de dois grupos, e os genótipos de Bourbon Amarelo (12, 13, 14 e 15), Bourbon Vermelho 2, Mundo

Tabela 5. Notas de vigor vegetativo de 20 genótipos de café, avaliados por quatro anos em cinco municípios do Estado de Minas Gerais ${ }^{(1)}$.

\begin{tabular}{lccccc}
\hline Genótipo $^{(2)}$ & $\begin{array}{c}\text { Campos } \\
\text { Altos }\end{array}$ & SAA $^{(3)}$ & Patrocínio & Lavras & $\begin{array}{c}\text { Três } \\
\text { Pontas }\end{array}$ \\
\hline BA1 & $7,50 \mathrm{~b}$ & $8,00 \mathrm{a}$ & $5,50 \mathrm{c}$ & $7,50 \mathrm{a}$ & $7,00 \mathrm{~b}$ \\
BA2 & $7,00 \mathrm{~b}$ & $7,50 \mathrm{a}$ & $5,00 \mathrm{c}$ & $7,50 \mathrm{a}$ & $7,50 \mathrm{~b}$ \\
BA3 & $7,50 \mathrm{~b}$ & $7,00 \mathrm{a}$ & $5,50 \mathrm{c}$ & $7,50 \mathrm{a}$ & $7,00 \mathrm{~b}$ \\
BA4 & $7,50 \mathrm{~b}$ & $6,50 \mathrm{~b}$ & $5,00 \mathrm{c}$ & $7,50 \mathrm{a}$ & $7,00 \mathrm{~b}$ \\
BA5 & $7,50 \mathrm{~b}$ & $7,00 \mathrm{a}$ & $5,00 \mathrm{c}$ & $7,00 \mathrm{a}$ & $7,50 \mathrm{~b}$ \\
BA6 & $7,50 \mathrm{~b}$ & $7,50 \mathrm{a}$ & $5,50 \mathrm{c}$ & $8,00 \mathrm{a}$ & $7,00 \mathrm{~b}$ \\
BA7 & $7,50 \mathrm{~b}$ & $8,00 \mathrm{a}$ & $5,50 \mathrm{c}$ & $8,00 \mathrm{a}$ & $7,50 \mathrm{~b}$ \\
BA8 & $7,50 \mathrm{~b}$ & $7,50 \mathrm{a}$ & $5,50 \mathrm{c}$ & $8,00 \mathrm{a}$ & $7,00 \mathrm{~b}$ \\
BA9 & $7,00 \mathrm{~b}$ & $7,50 \mathrm{a}$ & $5,50 \mathrm{c}$ & $7,50 \mathrm{a}$ & $7,50 \mathrm{~b}$ \\
BA10 & $6,00 \mathrm{~b}$ & $6,50 \mathrm{~b}$ & $5,50 \mathrm{c}$ & $7,50 \mathrm{a}$ & $7,50 \mathrm{~b}$ \\
BA11 & $6,50 \mathrm{~b}$ & $7,50 \mathrm{a}$ & $5,00 \mathrm{c}$ & $7,50 \mathrm{a}$ & $7,00 \mathrm{~b}$ \\
BA12 & $7,00 \mathrm{~b}$ & $9,00 \mathrm{a}$ & $5,50 \mathrm{c}$ & $8,00 \mathrm{a}$ & $8,50 \mathrm{a}$ \\
BV1 & $7,00 \mathrm{~b}$ & $7,50 \mathrm{a}$ & $5,50 \mathrm{c}$ & $7,00 \mathrm{a}$ & $7,00 \mathrm{~b}$ \\
BV2 & $7,50 \mathrm{~b}$ & $8,00 \mathrm{a}$ & $6,50 \mathrm{c}$ & $8,00 \mathrm{a}$ & $8,00 \mathrm{a}$ \\
BA13 & $9,00 \mathrm{a}$ & $7,50 \mathrm{a}$ & $6,00 \mathrm{c}$ & $8,00 \mathrm{a}$ & $9,00 \mathrm{a}$ \\
BA14 & $9,00 \mathrm{a}$ & $8,00 \mathrm{a}$ & $7,50 \mathrm{a}$ & $8,00 \mathrm{a}$ & $9,00 \mathrm{a}$ \\
BA15 & $8,00 \mathrm{a}$ & $7,50 \mathrm{a}$ & $7,50 \mathrm{a}$ & $8,00 \mathrm{a}$ & $8,50 \mathrm{a}$ \\
\hline Mundo Novo & $7,50 \mathrm{~b}$ & $8,50 \mathrm{a}$ & $7,00 \mathrm{~b}$ & $8,50 \mathrm{a}$ & $8,00 \mathrm{a}$ \\
Catuaí Vermelho ${ }^{(4)}$ & $7,50 \mathrm{~b}$ & $8,00 \mathrm{a}$ & $7,00 \mathrm{~b}$ & $7,50 \mathrm{a}$ & $7,50 \mathrm{~b}$ \\
Icatú Amarelo ${ }^{(4)}$ & $7,50 \mathrm{~b}$ & $7,50 \mathrm{a}$ & $7,50 \mathrm{a}$ & $7,50 \mathrm{a}$ & $8,50 \mathrm{a}$ \\
\hline
\end{tabular}

${ }^{(1)}$ Médias seguidas de letras iguais, nas colunas, não diferem pelo teste de Scott-Knott, a 5\% de probabilidade. ${ }^{(2)} \mathrm{BA}$, Bourbon Amarelo; BV, Bourbon Vermelho. ${ }^{(3)}$ SAA, Santo Antônio do Amparo. ${ }^{(4)}$ Testemunhas: Mundo Novo IAC 502/9, Catuaí Vermelho IAC 144 e Icatú Amarelo IAC 3282. 
Novo IAC 509/2 e Icatu Amarelo IAC 3282 formaram o grupo com maior nota de vigor. Apesar de terem sido detectadas diferenças entre os genótipos de Bourbon, de modo geral, estes não apresentaram elevadas notas de vigor, o que corrobora outros trabalhos realizados com a cultivar (Fazuoli et al., 2005; Moura et al., 2009).

Quando se trata de cafeeiros com potencial de produção de cafés especiais, é interessante a análise de peneira alta, pois essa característica proporciona maior uniformidade do lote a ser processado e influencia diretamente o aspecto físico do produto, o que é desejável, principalmente, para a utilização em máquinas de café expresso. Para esta característica, observou-se, em todos os locais estudados, a formação de pelo menos dois grupos, sendo que apenas os genótipos de Bourbon Amarelo 10 e 11, Bourbon Vermelho 2 e Icatu Amarelo IAC 3282 apresentaram maiores percentagens de peneira alta nos cinco locais estudados (Tabela 6). Vale ressaltar que o Bourbon Vermelho 2 também se destaca em produtividade e adaptabilidade. Em programas de melhoramento

Tabela 6. Percentagem de grãos peneira 16 e acima, de 20 genótipos de café, avaliados por quatro anos em cinco municípios do Estado de Minas Gerais ${ }^{(1)}$.

\begin{tabular}{|c|c|c|c|c|c|}
\hline Genótipo $^{(2)}$ & $\begin{array}{c}\text { Campos } \\
\text { Altos }\end{array}$ & $\mathrm{SAA}^{(3)}$ & Patrocínio & Lavras & $\begin{array}{c}\text { Três } \\
\text { Pontas }\end{array}$ \\
\hline & ---------- & 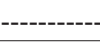 & $--(\%)--$ & 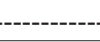 & --------- \\
\hline BA1 & $58,15 \mathrm{a}$ & $76,24 a$ & $56,87 \mathrm{~b}$ & $66,13 b$ & $67,03 b$ \\
\hline BA2 & $50,99 \mathrm{~b}$ & $72,87 b$ & $51,98 \mathrm{~b}$ & $63,50 \mathrm{~b}$ & $70,21 b$ \\
\hline BA3 & $62,32 \mathrm{a}$ & $80,30 \mathrm{a}$ & $55,51 b$ & $70,31 \mathrm{a}$ & $65,41 \mathrm{c}$ \\
\hline BA4 & $56,05 \mathrm{a}$ & $78,29 a$ & $56,69 \mathrm{~b}$ & $66,05 b$ & $67,3 \mathrm{~b}$ \\
\hline BA5 & $57,21 \mathrm{a}$ & $72,56 b$ & $52,54 b$ & $65,22 b$ & $70,63 b$ \\
\hline BA6 & $55,99 a$ & $73,89 b$ & $54,65 b$ & $64,54 b$ & $66,89 b$ \\
\hline BA7 & $57,60 \mathrm{a}$ & $77,18 \mathrm{a}$ & $53,79 b$ & $69,47 \mathrm{a}$ & $68,47 b$ \\
\hline BA8 & $62,08 \mathrm{a}$ & $71,12 b$ & $60,66 \mathrm{a}$ & $66,15 b$ & $67,21 \mathrm{~b}$ \\
\hline BA9 & $57,35 \mathrm{a}$ & $74,85 \mathrm{a}$ & $59,25 \mathrm{a}$ & $65,84 b$ & $74,51 \mathrm{a}$ \\
\hline BA10 & $62,56 \mathrm{a}$ & $80,10 \mathrm{a}$ & $59,08 \mathrm{a}$ & $73,77 \mathrm{a}$ & $73,62 \mathrm{a}$ \\
\hline BA11 & $60,14 \mathrm{a}$ & $80,04 a$ & $59,31 \mathrm{a}$ & $69,00 \mathrm{a}$ & $76,41 \mathrm{a}$ \\
\hline BA12 & $57,27 \mathrm{a}$ & $75,51 \mathrm{a}$ & $62,96 a$ & $65,26 \mathrm{~b}$ & $66,45 b$ \\
\hline BV1 & $59,93 \mathrm{a}$ & $75,47 \mathrm{a}$ & $51,40 b$ & $71,98 \mathrm{a}$ & $68,72 b$ \\
\hline BV2 & $57,23 \mathrm{a}$ & $79,52 \mathrm{a}$ & $64,01 \mathrm{a}$ & $71,07 \mathrm{a}$ & $77,21 \mathrm{a}$ \\
\hline BA13 & $46,79 b$ & $70,70 \mathrm{~b}$ & $45,62 \mathrm{c}$ & $64,54 b$ & $59,11 \mathrm{c}$ \\
\hline BA14 & $51,17 \mathrm{~b}$ & $73,81 b$ & $56,85 b$ & $66,78 \mathrm{~b}$ & $60,10 \mathrm{c}$ \\
\hline BA15 & $52,74 \mathrm{~b}$ & $72,57 \mathrm{~b}$ & $63,58 \mathrm{a}$ & $68,53 \mathrm{a}$ & $62,54 \mathrm{c}$ \\
\hline Mundo Novo ${ }^{(4)}$ & $58,55 \mathrm{a}$ & $81,89 \mathrm{a}$ & $67,88 \mathrm{a}$ & $72,12 \mathrm{a}$ & $72,84 b$ \\
\hline Catuaí Vermelho(4) & $59,87 \mathrm{a}$ & $77,61 \mathrm{a}$ & $61,54 a$ & $69,84 \mathrm{a}$ & $75,41 \mathrm{a}$ \\
\hline Icatú Amarelo $^{(4)}$ & $56,74 a$ & $65,56 \mathrm{~b}$ & $57,21 \mathrm{~b}$ & $61,20 \mathrm{~b}$ & $58,61 \mathrm{c}$ \\
\hline
\end{tabular}

${ }^{(1)}$ Médias seguidas de letras iguais, nas colunas, não diferem pelo teste de Scott-Knott, a 5\% de probabilidade. ${ }^{(2)} \mathrm{BA}$, Bourbon Amarelo; BV, Bourbon Vermelho. ${ }^{(3)}$ SAA, Santo Antônio do Amparo. ${ }^{(4)}$ Testemunhas: Mundo Novo IAC 502/9, Catuaí Vermelho IAC 144 e Icatú Amarelo IAC 3282. genético de cafeeiro, busca-se um ideótipo cujo desempenho abranja, além de outras características, alta produção e aumento de peneira (Ferreira et al., 2005).

Fazuoli et al. (2005), ao avaliar cultivares de Mundo Novo e Bourbon, também detectaram diferenças na percentagem de grãos de peneira alta entre os materiais, sendo que as progênies de Bourbon Amarelo e Bourbon Vermelho foram consideradas normais quanto à peneira média, porém, com valores menores do que os encontrados nas progênies de Mundo Novo, fato observado em apenas alguns genótipos no presente trabalho.

\section{Conclusões}

1. Há variabilidade genética dos cafeeiros dentro do grupo de genótipos de Bourbon Amarelo e Vermelho.

2. Os genótipos de Bourbon Amarelo e Vermelho apresentam produtividades satisfatórias em todos os locais.

3. O genótipo Bourbon Vermelho 2 foi o mais promissor quanto à adaptabilidade e à estabilidade, tendo-se destacado em todas as demais características de interesse agronômico avaliadas.

\section{Agradecimentos}

Ao Conselho Nacional de Desenvolvimento Científico e Tecnológico (CNPq), ao Consórcio de Pesquisa Café e à Fundação de Amparo à Pesquisa do Estado de Minas Gerais (Fapemig), pelo apoio financeiro ao projeto.

\section{Referências}

ANNICCHIARICO, P. Cultivar adaptation and recommendation from alfalfa trials in Northern Italy. Journal of Genetics and Breeding, v.46, p.269-278, 1992.

ANTUNES FILHO, H.; CARVALHO, A. Melhoramento do cafeeiro. XI - Análise da produção de progênies de híbridos de Bourbon Vermelho. Bragantia, v.16, p.175-195, 1957. DOI: 10.1590/S0006-87051957000100013.

BARROS, H.B.; SEDIYAMA, T.; MELO, A.V. de; FIDELIS, R.R.; CAPONE A. Adaptabilidade e estabilidade de genótipos de soja por meio de métodos uni e multivariado. Journal of Biotechnology and Biodiversity, v.3, p.49-58, 2012.

BOTELHO, C.E.; MENDES, A.N.G.; CARVALHO, G.R.; BARTHOLO, G.F.; CARVALHO, S.P. Seleção de progênies $\mathrm{F}_{4}$ de cafeeiro obtidas pelo cruzamento de Icatu com Catimor. Revista Ceres, v.57, p.274-281, 2010. DOI: 10.1590/ S0034-737X2010000300010. 
BRASIL. Ministério da Agricultura, Pecuária e Abastecimento. Instrução Normativa $\mathrm{n}^{\circ} 8$, de 11 de junho de 2003. Aprova o regulamento técnico de identidade e de qualidade para a classificação do café beneficiado grão cru. Diário Oficial [da] República Federativa do Brasil, 14 jun. 2003. Seção 1.

CARVALHO, A.; MÔNACO, L.C.; FAZUOLI, L.C. Melhoramento do cafeeiro: XL - estudos de progênies e híbridos de café Catuaí. Bragantia, v.38, p.202-216, 1979. DOI: 10.1590/S000687051979000100022 .

CARVALHO, G.R.; BARTHOLO, G.F.; MENDES, A.N.G.; NOGUEIRA, A.M.; MAGALHÃES, M.M. Seleção de progênies oriundas do cruzamento entre 'Catuaí' e 'Mundo Novo' em diferentes regiões do Estado de Minas Gerais. Bragantia, v.65, p.583-590, 2006. DOI: 10.1590/S0006-87052006000400008.

CILAS, C.; MONTAGNON, C.; BAR-HEN, A. Yield stability in clones of Coffea canephora in the short and medium term: longitudinal data analyses and measures of stability over time. Tree Genetics and Genome, v.7, p.421-429, 2010. DOI: 10.1007/ s11295-010-0344-4.

COMPANHIA NACIONAL DE ABASTECIMENTO. Acompanhamento da safra brasileira - café - safra 2013 segunda estimativa - maio/2013. Disponível em: $<$ http://www. conab.gov.br/OlalaCMS/uploads/arquivos/13_05_14_09_35_12_ boletim_cafe_maio_2013.pdf $>$. Acesso em: 10 jun. 2013.

CONDÉ, A.B.T.; COELHO, M.A. de; YAMANAKA, C.H.; CORTE, H.R. Adaptabilidade e estabilidade de genótipos de trigo sob cultivo de sequeiro em Minas Gerais. Pesquisa Agropecuária Tropical, v.40, p.45-52, 2010.

CORRÊA, L.V.T.; MENDES, A.N.G.; BARTHOLO, G.F. Comportamento de cafeeiro Icatu. Ciência e Agrotecnologia, v.30, p.618-622, 2006. DOI: 10.1590/S1413-70542006000400004.

CRUZ, C.D. Programa Genes: biometria. Viçosa: UFV, 2006. 382p.

FAZUOLI, L.C.; GUERREIRO FILHO, O.; SILVAROLLA, M.B.; MEDINA FILHO, H.P.; CARVALHO, A. Avaliação das cultivares de Mundo Novo, Bourbon Amarelo e Bourbon Vermelho de Coffea arabica L. em Campinas, SP. Bragantia, v.64, p.533-546, 2005. DOI: $10.1590 / \mathrm{S} 0006-87052005000400003$.

FERREIRA, A.; CECON, P.R.; CRUZ, C.D.; FERRÃO, R.G.; SILVA, M.F. da; FONSECA, A.F.A. da; FERRÃO, M.A.G. Seleção simultânea de Coffea canephora por meio da combinação de análise de fatores e índices de seleção. Pesquisa Agropecuária Brasileira, v.40, p.1189-1195, 2005. DOI: 10.1590/ S0100-204X2005001200005.

GICHIMU, B.M.; OMONDI, C.O. Early performance of five newly developed lines of Arabica coffee under varying environment and spacing in Kenya. Agriculture and Biology Journal of North America, v.1, p.32-39, 2010.
MENDES, F.F.; GUIMARÃES, L.J.M.; SOUZA, J.C.; GUIMARÃES, P.E.O.; PACHECO, C.A.P.; MACHADO, J.R. de A.; MEIRELLES, W.F.; SILVA, A.R. da; PARENTONI, S.N. Adaptability and stability of maize varieties using mixed model methodology. Crop Breeding and Applied Biotechnology, v.12, p.111-117, 2012. DOI: 10.1590/S1984-70332012000200003.

MOURA, W. de M.; LIMA, P.C. de; ASPIAZÚ, T.C.; SANTOS, J. dos; REIGADO, F.R.; SILVA, T.C. Desempenho de cultivares de café em cultivo orgânico no Município de Espera Feliz, MG safra 2009. Revista Brasileira de Agroecologia, v.4, p.2656-2659, 2009.

PEDRO, F.C.; GUIMARÃES, R.J.; CARVALHO, G.R.; BOTELHO, C.E.; REZENDE, J.C. de; CARVALHO, A.M. Comportamento agronômico de progênies F4 de cafeeiros oriundos do cruzamento entre os cultivares Mundo Novo e Catuaí. Revista Ceres, v.58, p.139-150, 2011. DOI: 10.1590/ S0034-737X2011000300011.

PEREIRA, H.S.; ALMEIDA, V.M. de; MELO, L.C.; WENDLAND, A.; FARIA, L.C. de; DEL PELOSO, M.J.; MAGALDI, M.C. de $\mathrm{S}$. Influência do ambiente em cultivares de feijoeiro-comum em cerrado com baixa altitude. Bragantia, v.71, p.165-172, 2012. DOI: $10.1590 / \mathrm{S} 0006-87052012005000024$.

RAMALHO, M.A.P.; FERREIRA, D.F.; OLIVEIRA, A.C. de. Experimentação em genética e melhoramento de plantas. Lavras: UFLA, 2000. 326p.

RAMOS, L.M.; SANCHES, A.; COTES J.M.; CARGNELUTTI FILHO, A. Adaptabilidade e estabilidade do rendimento de genótipos de arroz, mediante duas metodologias de avalição na Colombia. Acta Agronómica, v.60, p.39-49, 2011.

SEVERINO, L.S.; SAKIYAMA, N.S.; PEREIRA, A.A.; MIRANDA, G.V.; ZAMBOLIM, L.; BARROS, U.V. Associações da produtividade com outras características agronômicas de café (Coffea arabica L. "Catimor"). Acta Scientiarum. Agronomy, v.24, p.1467-1471, 2002.

SILVEIRA, L.C.I. da; KIST, V.; PAULA, T.O.M. de; BARBOSA, M.H.P.; OLIVEIRA, R. A. de; DAROS, E. Adaptabilidade e estabilidade fenotípica de genótipos de cana-de-açúcar no estado de Minas Gerais. Ciência Rural, v.42, p.587-593, 2012. DOI: 10.1590/S0103-84782012000400002.

STEEL, R.G.D.; TORRIE, J.K. Principles and procedures of statistics: a biometrical approach. $2^{\text {nd }}$ ed. Tokyo: McGraw-Hill, 1980. 633p.

VASCONCELOS, E.S. de; REIS, M.S.; CRUZ, C.D.; SEDIYAMA, T.; SCAPIM, C.A. Adaptability and stability of semilate and late maturing soybean genotypes in Minas Gerais state. Acta Scientiarum. Agronomy, v.32, p.411-415, 2010. DOI: 10.4025/ actasciagron.v32i3.8249.

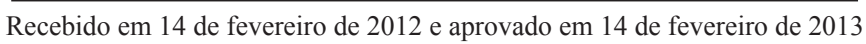

